\title{
A Discrete Multiphase Approach For The Infiltration Of A Nanofluid Around A Staggered Microfiber Matrix
}

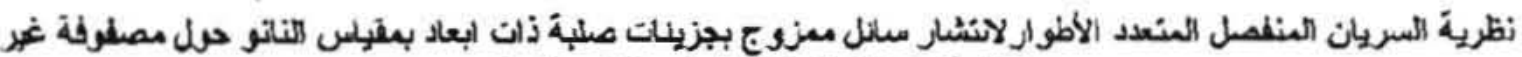

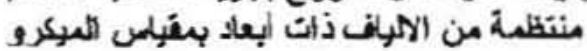

Ahmed Elgafy,

Associate Research Engineer.

University of Dayton Research Instuite, 300 College Park, Dayton, OH, 45469 USA

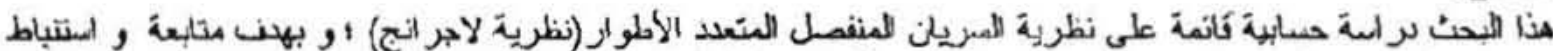

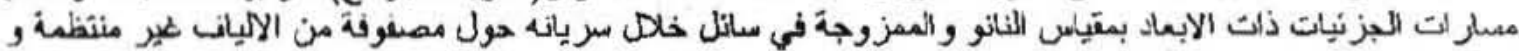

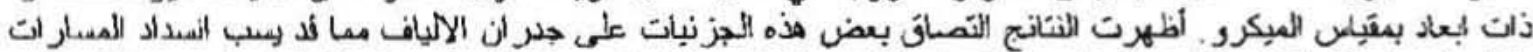

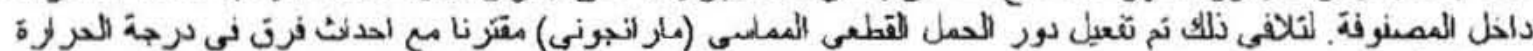

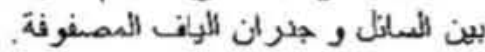

\section{Abstract}

In the present work a numerical study based on discrete multiphase approach, Lagrangian approach, is proposed to investigate and predict the trajectories of nanoparticles-filled fluid (nanofluid) infiltrated around a staggered microfiber matrix. The trajectory of the nanoparticle has been predicted by integrating the force balance on it in a Lagrangian reference frame during its motion through the fluid. The governing integral equations for the conservation of mass, momentum and energy have been solved in a segregated numerical fashion by a ControlVolume-Based Finite-Element Method. The nanoparticles trajectories and their interactions with fluid flow and microfiber walls bave been predicted showing undesirable sticking tendency of the nanoparticles on the microfiber walls. To avoid this tendency, the contribution of Marangoni shear stress has been introduced coupled with a tempcrature difference between the fluid flow and the microfiber walls.

Keywords: Nanoparticles - Lagrangian approach - Trajectories - Solid/liquid interaction

\section{Introduction}

Nanoparticle flows have been emerging in numerous biological, and scientific applications, such as blood clogging and cell transport in arteries and veins, drug and gene delivery, and manufacturing of nanocomposites. Accordingly, many efforts are focused on the ability to create novel composites using nanocomposite materials as new matrices and to model various composite processes through numerical methods, Milton [1]; Kim et al. [2]; and Chen et al. [3]. In particular, enhancing and predicting the thermal performance of fluids and composites using of nanoparticle additives have been considered by many researchers Lee \& Choi [4]; Xuan \& Li [5];
Khanafer et al. [6]; Elgafy and Lafdi [7]. The presence of nanoparticles in fluids increases their effective thermal conductivities and consequently enhances their heat transfer characteristics Choi 1995 [8]; Lee et al. 1999 [9]; Xuan \& Roetzel 2000 [10]; Eastman et al. 2001 [11]; Yu \& Choi 2003 [12]. Besides thermal properties, nanoparticles additives may enhance mechanical properties of a given new system. In theory, carbon based nanoparticles could enhance both chemical and physical properties of composites Mauran et al. 1993 [13]; Py et al. 2001 [14]; Fukai et al. 2000 [15]. Moreover, fluids that contain nanoparticles additives (nanofluids) have a distinctive characteristic, which is quite different from those of traditional solid- 
liquid mixtures in which millimeter and/or micrometer-sized particles are involved. Such particles could create pressure doop due to settling effects. In contrast, the nanofluids exhibit little or no penalty in pressure drop when flowing through the passages, Khanafer et al. [6]. Nevertheless, the possibility of clogging the micro-scale passages still exist due to intenctions beiween the nanoparticle additives and the passage walls. This trend is magnified in case of some composite processes because of high resin viscosity and low preform permeability Conversely, and from fluid mechanics point of view, the flow of nanoparticlo-filled fluids, nanofluids, is categorized as multiphase flow. Simulation and prediction of the characteristics of such flows could be very complex. Regardless of this complexity. significant progress has been made in different areas of multiphase Computational Fluid Dynamics, CFD. Wachem and Almsted [16] introduced different physical models for CFD predictions of multiphase flows while Klainetreuer [17] introduced their nunerical solution tools. It was depicted that there are two different basic approaches for studying this kind of flows. The first one is the continuum model, Eulerian approach, in which both the fluid phase and the solid phase are treated as continuous media, Jackson [18]. The second approsech is the discrete particle model, Lagangian approach, which treats the solid phase as separate particles that interact with the flow and traces the position and velocity of all the particles by solving Lagnangian equation of motion. The fluid phase is still considered as a continuum phase, and the effects of the solid phases are included by adding mass and force terms into the continuity and motnentum equations of the fluid, Patankar and Joseph [19]. For both approaches, many other considerations should be taken in case of submicron and nano scales particles, Gidaspow [20]. As it is scen, Lagrangian approsch is appropriate for modeling multiphase problems that aim 10 study the behavior and interaction of the solid phase individuals, particles, with the continuous phase, fluid.

In the present paper, a two-dimensional simulation model based on Lagrangian multiphase approach for nanoparticle-filled fluid "nanofluid", which flows around staggered mictofiber matrix, is introduced to investigate and predict the nanoparticles trajectories and their internctions with the fluid flow and the microfiber walls. Preventing any potential sticking of the nanoparticles on the microfiber walls is also a vital goal for the preseat study.

\section{Numerical formulafion}

\subsection{System configuration und physieal demain}

A nanofluid, which is composed of spherical carbon nanoparticles dispersed homogenously into a fluid, is infiltrated into a two-dimensional carbon microfiber matrix as shown in Fig. 1a. The mierofibers have the same diameler D and afe arranged in a staggered configuration with a separate distance $T=D$ from all directions. The used nanofluid is assumed to be Newtonian, incompressible, laminar and its viscosity is not affected by small amount of nanoparticle additives. The inflination process is supposed to occur during a short period (in second scale) while the second phase (nanoparticle) is sufficiently dilute that particle-particle interactions are negligible. A unit cell is taken as a representative unit cell for the system as shewn in Fig. 1b, which illustrates that one-half of each side-by-side microfiber is only taken for symmetrical considerations, while the third microfiber. which is in a staggered orientation with the other two microfibers, is completely considered. As a case of study, carbon microfibers (8 $\mu \mathrm{m}$ in diameter) are considered, and a unit cell ( $16 \mu \mathrm{m} \times 38 \mu \mathrm{m})$ 
represents the model. The carbon nanoparticles have a diameter of $100 \mathrm{~nm}$. The thermo-physical properties of used fluid and solid particles (carbon nanoparticles) are listed in table 1.

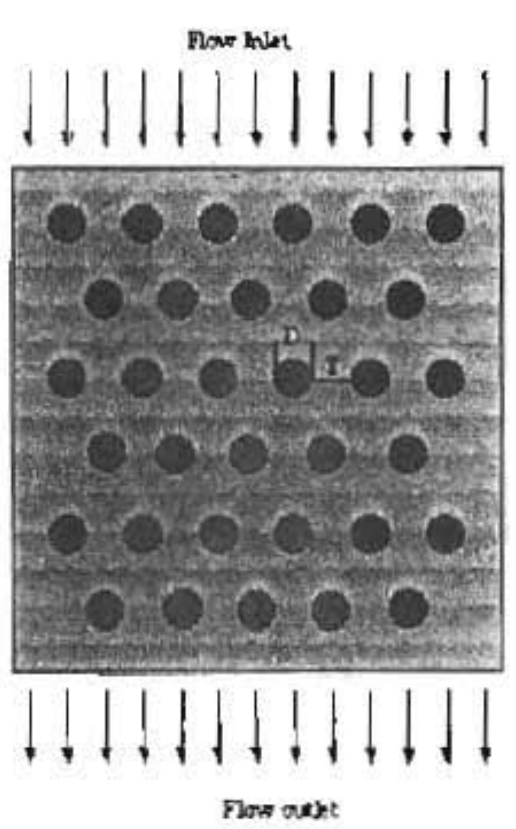

Fig, Ia Carbon mierofibers matrix configuration

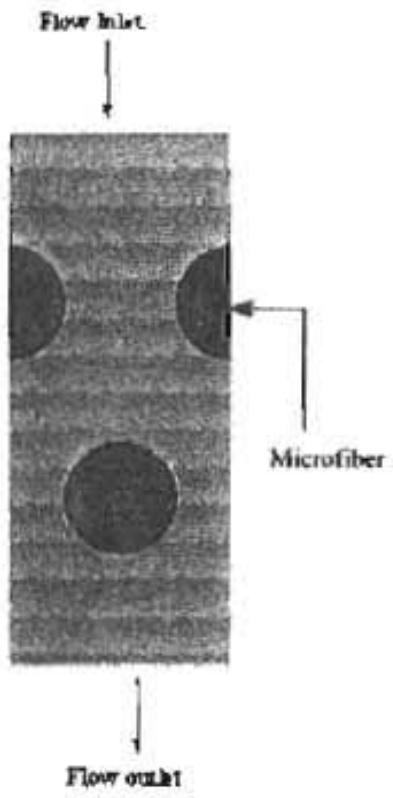

Fig. Ib Model's represenlative unit cell
Table 1: Thermophysical properties of the used fluid and solid nanoparticles

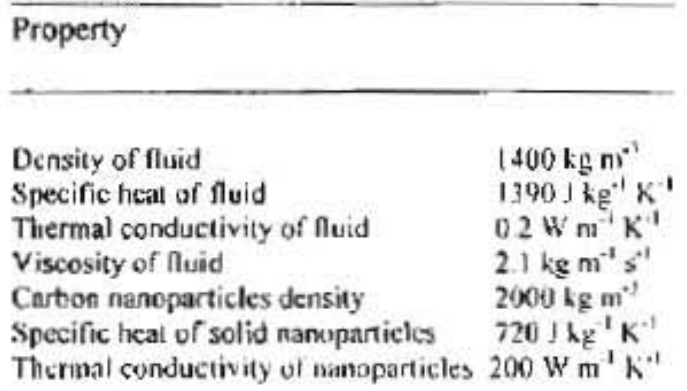

\subsection{Model description and, governing equations}

A two-dimensional simulation model based on Lagrangian multiphase approach for nanofluid, which is infiltrated around staggered microfiber matrix, is introduced to investigale and predict the nanoparticles trajectories and their interactions with the fluid flow and the microfiber walls. In this section, the governing equations of conservation of mass, momentum and energy of the system are introduced as well as the calculations of the nanoparticles trajectories coupling with the continuous phase, fluid.

\subsubsection{Governing equations}

In the present model, the fluid flow is considered as a two dimensional, stcady laminar flow and the governing equations for conservation of mass, momentum and energy for two-dimensional axisymmetrical geometries, could be taken as follows:

\subsubsection{Continuity equation}

$\frac{\partial \rho}{\partial r}+\frac{\partial}{\partial x}\left(\rho v_{z}\right)+\frac{\partial}{\partial r}\left(\rho v_{r}\right)+\frac{\rho v_{r}}{r}=S_{m}$

where, $x$ is the axial coordinate, $r$ is the radial coordinate, $\rho$ is the fluid density, $v_{x}$ is the axial velocity, and $v_{r}$ is the radial velocity and the source term $S_{m}$ is the mass added to the continuous phase; resin. from the second phase; carbon nanoparticles. 
M. 59 Ahmed Elgafy.

\subsubsection{Momentum equations}

$$
\begin{aligned}
& \frac{\partial}{\partial t}\left(\rho v_{n}\right)+\frac{1}{r} \frac{\partial}{\partial x}\left(r \rho v_{1}, v_{n}\right)+\frac{1}{r} \frac{\partial}{\partial r}\left(r \rho v_{,} v_{3}\right)= \\
& -\frac{\partial p}{\partial x}+\frac{1}{r} \frac{\partial}{\partial x}\left[r \mu\left(2 \frac{\partial v_{A}}{\partial x}-\frac{2}{3}(\nabla \cdot \bar{v})\right)\right] \\
& +\frac{1}{r} \frac{\partial}{\partial r}\left[r \mu\left(\frac{\partial v_{v}}{\partial r}+\frac{\partial v_{c}}{\partial x}\right)\right]+F \text {, }
\end{aligned}
$$

and

$\frac{\partial}{\partial t}\left(\rho v_{t}\right)+\frac{1}{r} \frac{\partial}{\partial x}\left(r \rho v_{t} v_{t}\right)+\frac{1}{r} \frac{\partial}{\partial r}\left(r \rho v_{r} v_{l}\right)=$

$-\frac{\partial p}{\partial r}+\frac{1}{r} \frac{\partial}{\partial r}\left[r \mu\left(2 \frac{\partial v}{\partial r}-\frac{2}{3}(\nabla \cdot \bar{v})\right]\right.$

$+\frac{1}{r} \frac{\partial}{\partial r}\left[r \mu\left(\frac{\partial v_{v}}{\partial x}+\frac{\partial v_{f}}{\partial r}\right)\right]$

$-2 \mu \frac{v_{r}}{r^{2}}+\frac{2}{3} \frac{\mu}{r}(\nabla \cdot \bar{v})+F$,

where $p$ is the pressure, $\mu$ is the fluid viscosity, $F$ is the external body forces that arise from inieraction with the second phase, nanoparticles, and

$$
\nabla \cdot \vec{\nabla}=\frac{\partial v_{f}}{\partial x}+\frac{\partial v_{t}}{\partial r}+\frac{v_{f}}{r}
$$

\subsubsection{Energy equation}

The energy equation for the present model is taken as given by Shin and Jurec $[21]$ in the following form

$$
\frac{\partial(p c T)}{\partial t}+\nabla(\rho c T \nabla)=\nabla(k \nabla T)+S_{k}
$$

which, could be written as

$$
\begin{aligned}
& \frac{\partial(p c T)}{\partial t}+\frac{\partial}{\partial x}\left(\rho c T v_{t}\right)+\frac{\partial}{\partial r}\left(\rho c T v_{t}\right)+ \\
& \rho c T \frac{v_{t}}{r}=\nabla \cdot(k \nabla T)+S_{h} \ldots \ldots \ldots \ldots(6)
\end{aligned}
$$

where, $\mathrm{c}$ and $\mathrm{k}$ are the fluid specific heat and thermal diffusivity respectively, $T$ is the temperature and $S_{6}$ is the heating source term.

\subsection{Nanoparticles trajectory calculations}

\subsubsection{Equation of motion}

The equation of motion for a representative particle in Lagrangian reference frame could be taken ss given by Kleinstreuer [17] in the following form:

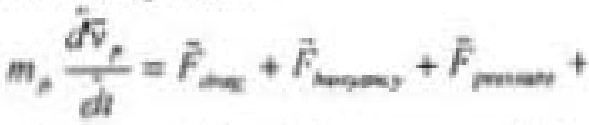

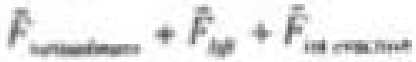

where. $m_{p}$ and $v_{p}$ are the particle mass and velocity respectively, and $\dot{j} / d t=\partial / \partial t+\hat{v},-\nabla$, ie, a time derivative following the moving particle

\subsubsection{Visceus drag force, $\hat{F}_{\text {the }}$}

The viscous drag force acting on the particle given by Kleinstreuer [17] is in the following form:

$$
\vec{F}_{\text {tas }}=m_{p} C, \frac{\hat{d}\left(\bar{v}_{f}-\vec{v}_{p}\right)}{d t}
$$

where, $\vec{v}_{f}$ is the fluid velocity and $C_{d}$ is the drag coefficient for submicron and nano seales particles given by Ounis et al. [22] and it has the form:

$$
C_{n}=\left(\frac{18 \mu}{C_{v} \rho_{p} d_{p}^{2}}\right)
$$

where, $\mu$ is the fluid viscosity, $\rho_{p}$ and $d_{p}$ are the particle density and diameter respectively, and $C_{t}$ is the Cunningham slip correction factor to Stokes' drag law, which can be computed from the following relation

$$
C_{r}=1+\frac{2 \lambda}{d_{r}}\left(1.257+0.4 e^{\left(11 \frac{\sigma_{e}}{2 \lambda}\right)}\right) \ldots(10)
$$

where, $\lambda$ is the molecular mean free path, Kleinstreucr [17].

\subsubsection{Buoyancy force, $\vec{F}_{\text {haumy }}$}

The net buoyant force acting on the particle is estimated from the following relation

$$
\tilde{F}_{\text {havency }}=m_{p}\left(1-\frac{\rho_{f}}{\rho_{p}}\right) \bar{k}
$$


where, $\rho_{f}$ is the fluid density and $g$ is the gravity.

\subsubsection{Pressure force, $\bar{F}_{\text {precure }}$}

There pressure force acting on the particle is due to dynamic pressure and shear stress gradients, and its formula is given by Kleinstreuer [17] in the following form

$\vec{F}_{\text {presiure }}=-V_{p}(\nabla p+\nabla \cdot \overline{\bar{\tau}})$

where, $V_{p}$ is the particle volume, $p$ is the pressure and $\overline{\bar{\tau}}$ is the shear stress.

\subsubsection{Virtual mass force, $\bar{F}_{\text {vrruatmass }}$}

The virtual mass force is the force required to accelerate the fluid surrounding the particle and it is taken as given by Kleinstreuer [17] in the form

$$
F_{\text {viruaimass }}=\frac{1}{2} m_{r} \frac{\rho_{f}}{\rho_{r}} \frac{\hat{d}\left(\bar{v}_{f}-\bar{v}_{p}\right)}{\hat{d}_{l}} \ldots
$$

\subsubsection{Lift force, $\vec{F}_{\text {ligh }}$}

The lift force due to shear, Saffman lift force, is taken as given by $\mathrm{Li}$ and Ahmadi [23] in the following form:

$$
F_{i}^{i, n}=5.2 m_{p} \frac{\rho_{f} v_{j}^{0.5} \varepsilon_{l y}\left(v_{j}^{f}-v_{j}^{p}\right)}{\rho_{p} d_{p}\left(\varepsilon_{l k} \varepsilon_{k l}\right)^{1 / 4}}
$$

where, $v_{f}$ is the fluid kinematic viscosity and $\varepsilon_{i j}$ is the deformation-rate tensor, which has the following form

$$
\varepsilon_{y}=\frac{1}{2}\left(\frac{\partial v_{i}}{\partial x_{i}}+\frac{\partial v_{1}}{\partial x_{1}}\right)
$$

\subsubsection{Interaction force, $\vec{F}_{\text {int craction }}$}

In the present model we will consider the interaction forces due to Brownian motion effects. The Brownian force was modeled by $\mathrm{Li}$ and Ahmadi [24] as a Gaussian white noise random process where the amplitudes of the components at every time step, $\Delta t$ :
$F_{1}^{\text {Rrown }}=G_{t}^{m_{r}}\left(\frac{\pi S_{0}}{\Delta t}\right)$

where, $G_{i}$ are the zero-mean, unit varianceindependent Guassian random numbers; and $\mathrm{S}_{0}$ is the spectral intensity;

$$
S_{n}=216 \frac{v_{f} \sigma T}{\pi^{2} \rho_{f} d_{\rho}^{s}\left(\frac{\rho_{\rho}}{\rho_{f}}\right)^{2} C_{c}}
$$

Where $T$ is the absolute temperature of the fluid, $\sigma$ is the Stefan - Boltzman constant, $\mathrm{C}_{c}$ is the Cunningham slip correction factor, equation (10).

\subsection{Heat transfer calculations}

To relate the particle temperature, $T_{\rho}(l)$, to the convective heat transfer a heat balance was performed and the following relation was utilized:

$$
m_{p} c_{p} \frac{d T_{p}}{d t}=h A_{p}\left(T_{f}-T_{n}\right)
$$

where: $c_{p}$ and $A_{p}$ are the heat capacity and the surface area of the particle, $T_{f}$ is the local temperature of the continuous phase, fluid, $h$ is the convective heat transfer coefficient.

As the particle trajectory is computed, equation (18) is integrated to obtain the particle temperature at the next time value, yielding:

$T_{p}(t+\Delta t)=T_{f}+\left[T_{p}(t)-T_{j}\right] e^{-p_{r} \Delta}$

Where; $\Delta t$ is the integration time step and

$$
\beta_{p}=\left(\frac{A_{p} h}{m_{p} c_{p}}\right)
$$

The heat transfer coefficient, $h$, is evaluated using the following correlation given by Incropera and DeWitt [25];

$$
N u=\frac{h d_{p}}{k_{s}}=2.0+0.6 \mathrm{Re}_{d}^{1 / 2} \mathrm{Pr}_{f}^{1 / 3}
$$

Where; $k_{f}$ and $P r_{f}$ are is thermal conductivity and Prandts number of the continuous phase, fluid, respectively and $R_{d}$ is Reynolds number based on the particle diameter and the relative velocity. 
M. 61 Ahmed Elgaly.

\subsection{Numerical solution}

The numerical analysis has been performed using Fluent as follows. The momentum and heat exchanges from the continuous phase, fluid, to the discrete phase, nanoparticle, are computed by examining the change in momentum and thermal energy of the nanoparticle as it passes through each control volume in the model. These momentum and heat exchanges appear as momentum sink and source or sink energy in the continuous phase momentum and enetgy balances respectively in any subsequent calculations of the continuous flow field. During the motion of the particles, it is assumed that they do not exchange mass or heut by radiation with the continuous phase and they do not participste in any chemical reaction. The governing integral equations for the conservation of mass, momentum and enerzy for the model are solved in a segregated numerical fashion by a Control-Volame. Based Finite-Element Method, Patankar [26].

\subsection{Grid size}

A grid size of $50 \times 120$ is applied on the unit cell illustrated in Fig. 1b. To lest and assess grid independence of the solution scheme, the grid size has been changed up to $50 x$ 120 , and it has been found that this grid size is sufficient for the present case of study.

\subsection{Initial and boundary cooditions}

The numerical model has been solved under the following assumptions and boundary and initial conditions: the thermophysical propertics of the used fluid are introduced at a temperature level of $300^{\circ} \mathrm{K}$. The flow inlet is defined by its velocity components, while the flow outlet is defined by its outlet pressure. The iniet velocity of the flow is assumed to be normal to the top boundary of the cell and has a magnitude of $0.05 \mathrm{~m} / \mathrm{s}$ with the gravity direction and the flow outlet pressure is assumed to have the almospheric pressure value Since the flow thermal solution is assumed to be periodic; periodic boundary conditions are applied on the side boundaries of the flow inlets and outlets. The carbon microfiber walls are assumed to have a temperature of $300^{\circ} \mathrm{K}$ with no internal heat generation and no slip boundary condition. A condition of gravitational acecleration is also considered. Inert spherical carbon particles, which have a diameter of $100 \mathrm{~nm}$, are injected through the fluid fow with different loads to investigate and predict the nanoparticles trajectories and their interactions with the fluid flow and the microfiber walls.

\section{Results}

The present model has been solved first for the flow of the sole fluid without the injection of the nanoparticles to study the flow characteristics and its interaction with the microfiber walls. As the main issue of the present study, the model has been solved with the injection of inert spherical carbon nanoparticles with fluid flow from different locations with difletent loads to investigate and predict nanoparticles trajectorics and their interactions with the fluid flow and the microfiber walls.

\subsection{Single fluid flow characteristics}

The predicted static pressure contours for single fluid are illustrated in Fig. $2 \mathrm{a}$. The figure shows that the maximum static pressure is achieved over the upper ends of the upper two mierofibers due to their reactions against the duid head. Also from this figure, one can sec that the static pressure at the upper half of the cell decreases gradually towards the radial direction, $r$, and it has a minimum value at the oell center between the side-by-side microfibers. Conversely, the static pressure decreases sharply from the upper end to the lower end along the axial direction, $x$, until it 
reaches a constant value at the lower half of the cell after passing the third microfiber.

The predicted dynamic pressure contours for single fluid are shown in Fig. 2b. Unlike the static pressure, the dynamic pressure increases from the wall sides toward the radial direction until it reaches maximum values at the center between the side-by-side microfibers. On the other hand, the dynamic pressure has less value around the third microfiber.

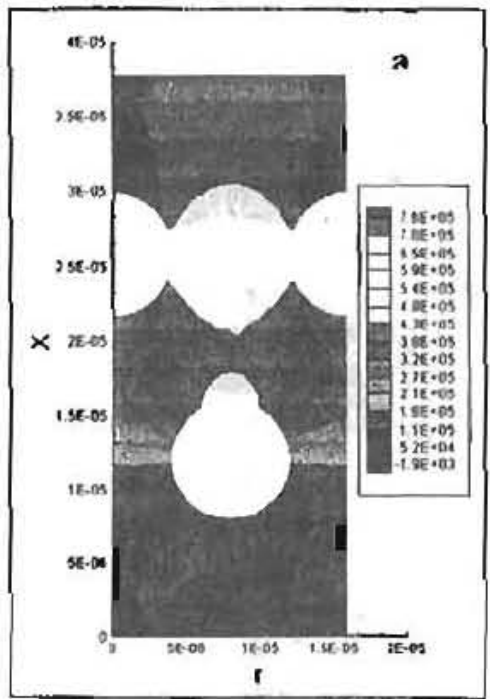

Fig. 2a Static pressure contours for single fluid in (Pa)

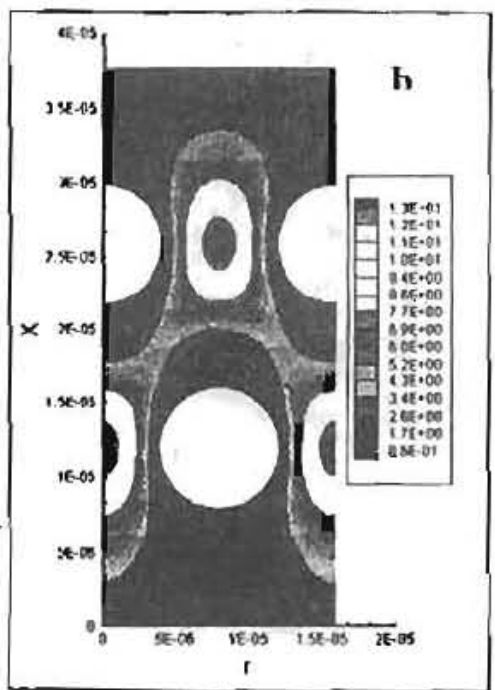

Fig.2bDymamic pressure contours for single fluid in (Pa)
The predicted Reynolds number contours for the flow of single fluid are presented in Fig. 3a. The figure shows that the Reynolds number has minimum values around all microfiber walls as a result of high friction. The friction decreases gradually toward the center of the cell at the radial direction between the side-by-side microfibers where microfiber walls-fluid interaction diminished, while this friction decreases gradually around the third microfiber. Similarly, the shear stress is much higher at microfiber wallsfluid interface, Fig. $3 b$.

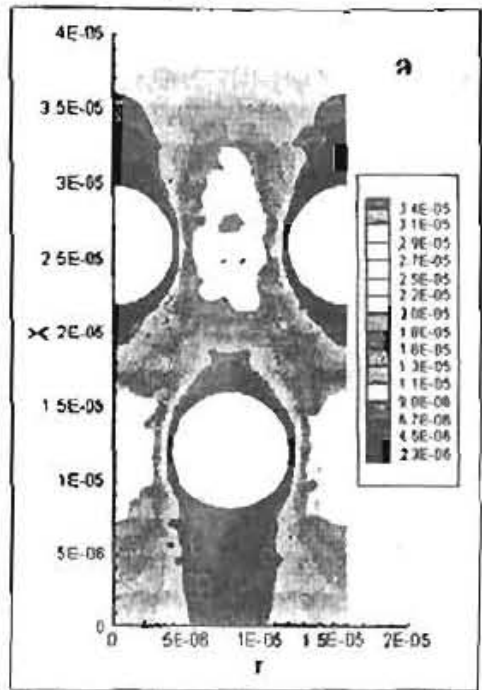

Fig. 3a Reynolds number contours for single fluid

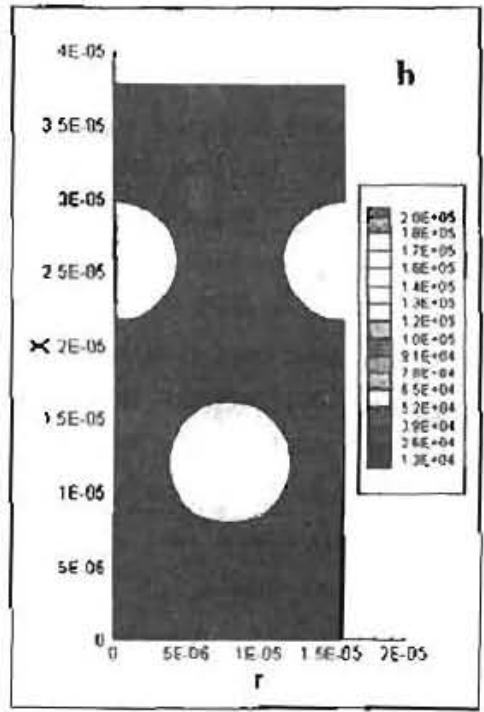

Fig. $3 b$ Walls shear stress contours for single fluid, ( $\mathrm{Pa}$ ). 


\section{63 Ahmed Elgafy}

\subsection{Nanoparticles trajecteries}

The present model has been performed and solved for the injection of inert spherical carbon particles, which have a diameter of $100 \mathrm{~nm}$, through the fluid flow from different locations of the system entrance with different nanoparticle load ratios. The nanoparticle trajectories for each case are predicted as well as their interactions with fluid flow and carbon microfiber walls.

\subsubsection{Injection of nanoparticles with differeat load ratios}

One nanoparticle has been injected through the fluid flow from the mid point of the unit cell entrance as shown in Fig. 4. The figure shows that the particle has moved directly between the two side-by-side microfibers until it has been trapped on the wall of the third microfiber. From this figure one can conclude that the effect of the two side-byside microfibers on the nanoparticle motion has been eliminated by each other. Nevertheless, the particle could not survive when it has moved through the high friction zone around the third microfiber wall. On the other hand, when three nanoparticles have been injected between locations $P(5 \mathrm{c}$ $06,3.7 e-05)$ and $P(1.55 e-05,3.7 e-05)$, which two of the particles are very close to the walls of the two side-by-side microfibers, all the particles have been trapped on the three microfiber walls due to the high friction effect, Fig. 5. The injection of nine nanparticles between locations $\mathrm{P}(\mathrm{Se}-06,3,7 \mathrm{e}$ 05) and $P(1.55 e-05,3.7 e-05)$ is presented in Fig. 6. From the figure one can notice again that the nanoparticles, which have moved near the microfiber walls, have been trapped on their surfaces; while the other particles have the tendency to track the flow pattern around the microfibers walls.

The undesirable sticking tendency of the nanoparticles on the microfiber walls during the flow process, could occur because the interfacial fluid layers around the microfibers exhibit high friction. This friction tends to reduce the flow velocity, which may force some of the nanoparticles to stick on the microfiber walls and after sometime, perhaps, the flow passages will be blocked.

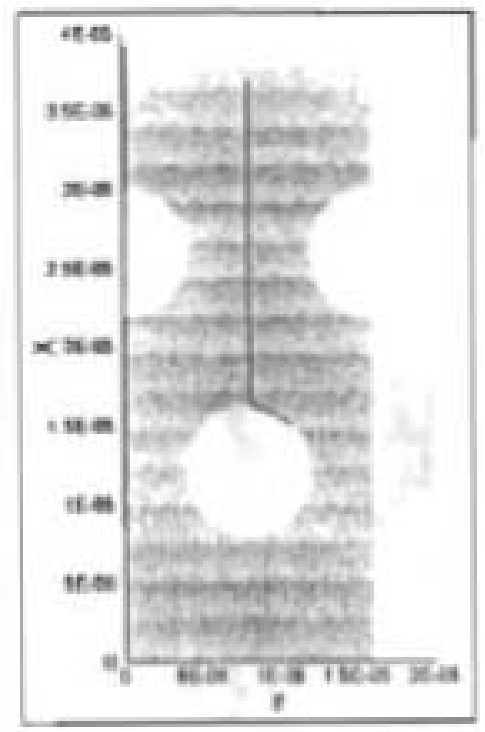

Fig. 4 One particle injected from the mid point, P(Be-06, 3.7e-05)

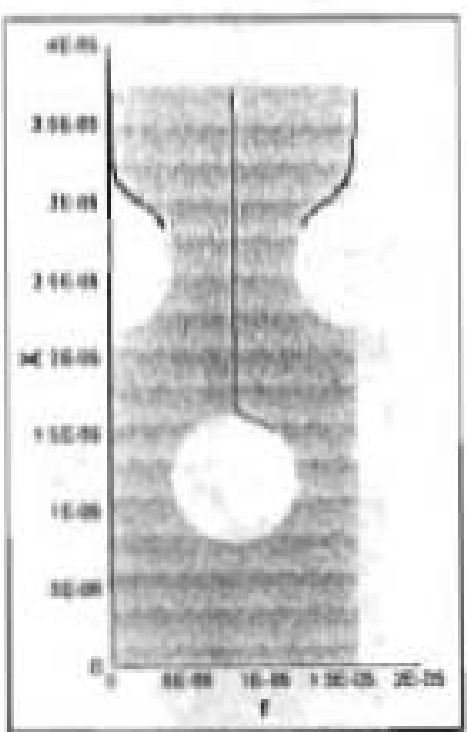

Phe 5 Three particles injected between two locations, $P(5 e-06,3,7 e-05)$ and $P(1.55 e-05,3,7 e-05)$ 


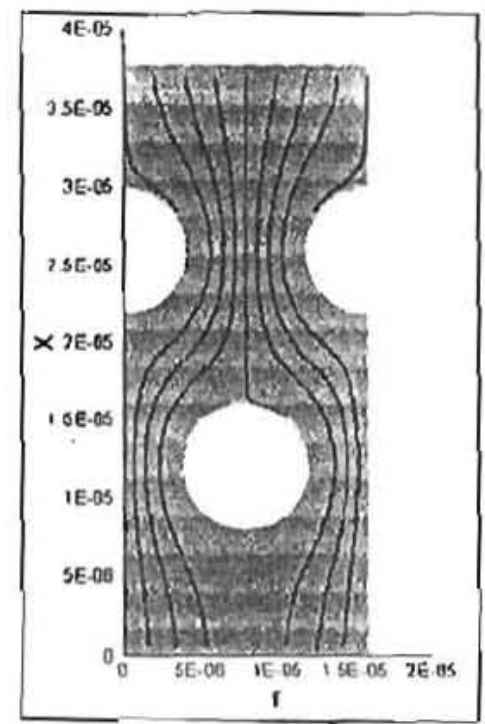

Fig.6 Nine particles injected between two locations, $\mathrm{P}(\mathrm{Se}-06,3.7 \mathrm{e}-05)$ and $\mathrm{P}(1.55 \mathrm{e}-05,3.7 \mathrm{e}-05$

\subsection{Contribution of Marangoni shear} stress:

To prevent any potential sticking of the injected particles on the microfiber walls during the flow process; the contribution of Marangoni shear stress could be introduced coupled with a temperature difference between the fluid flow and the microfiber walls. As reported by Schwartz [27], the lemperature-induced surface stress will contribute to the flow within the liquid laycr. These thermocapillary effects give rise to various gravity independent phenomena including convective flows, interface distortions as well as interface ruplure. Thermocapillary flows are driven by the imbalance of tangential stress on the interface caused by temperature dependence of surface tension, Jiang \& Floryan [28]. This tangential stress is called Marangoni shear stress, and it has the following relation:

$\tau_{\text {Morongoni }}=\frac{\partial \sigma}{\partial T} \cdot \frac{\partial T}{\partial s}$ where $\frac{\partial \sigma}{\partial T}$ is the surface tension temperature coefficient and $\frac{\partial T}{\partial s}$ is the tangential vector of local free surface.

As it is seen from the definition of Marangoni shear stress, it is a combination between a surface tension and a temperature gradient, which offers a wealth of possible responses. To introduce the energy imbalance lechnique, the model has been performed and solved including the Marangoni shear stress contribution coupled with a temperature difference between the fluid flow and the microfiber walls. Many temperature difference levels have been applied while a fixed value of the surface tension temperature has been introduced, $\partial \sigma / \partial T=0.4 \mathrm{~N} / \mathrm{m} . \mathrm{K}$.

The predicted temperature contours for various temperature difference levels for the sole flow are shown in Fig.7. As shown from the figure, temperature gradient has been built between the carbon microfibers walls and the fluid flow and this difference is getting stronger in the case of higher temperature difference levels.

The predicted Reynolds number contours for the sole flow after applying the new technique for different temperature levels are shown in Fig. 8. In general, one can see that introducing the new technique creates convective currents around the carbon microfiber walls, which cause kind of vortices around them. These vortices are forcing the flow to move away from the carbon microfiber walls and they are stronger in the case of higher temperature difference levels. 
M. 0.5 Ahmed Elgally.
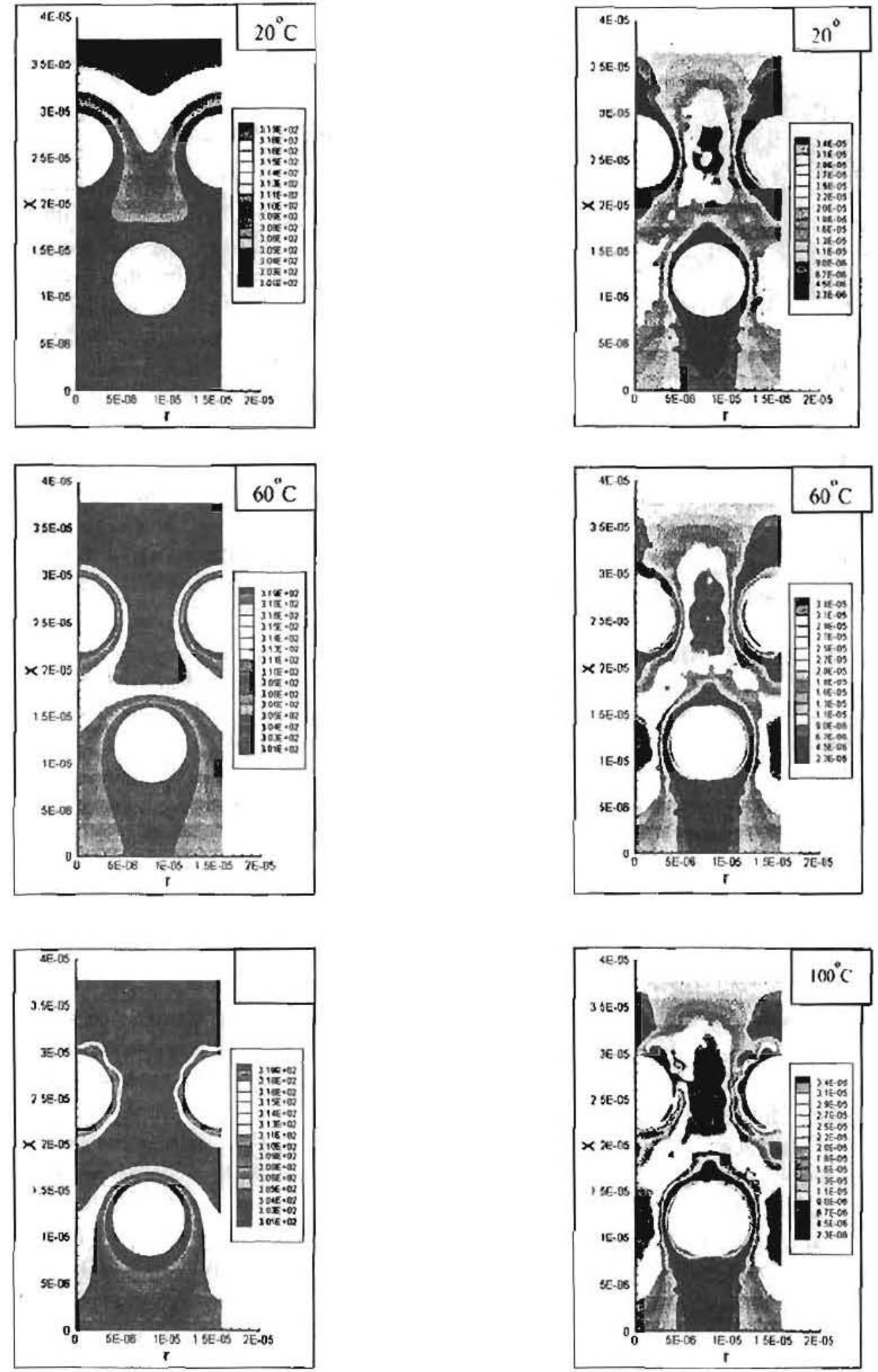

Fig. 7 Temperature contours for a temperature difference, $\Delta \mathrm{T}$, of 20,60 and $100^{\circ} \mathrm{C}$.

Figure. 8 Reynolds number contours for a temperature difference, $\Delta \mathrm{T}$, of 20,60 and $100^{\circ} \mathrm{C}$. 
The predicted results for the trajectories of one and multiple nanoparticles after introducing the energy imbalance techniques are shown in Fig. 9 for a temperature difference level of $60^{\circ} \mathrm{C}$. The figures show that the nanoparticles, which were trapped on the microfiber walls before introducing the technique, are moving away from the microfiber walls and they do not adhere on them.

\section{Conclusions}

A two-dimensional simulation model based on Lagrangian multiphase approach for - nanoparticle-filled fluid "nanofluid" which flows around a staggered microfiber matrix, has been introduced. The nanoparticles trajectories and their interactions with fluid flow and microfiber walls have been investigated numerically showing undesirable sticking tendency of the nanoparticles on the microfiber walls. To prevent this tendency, the contribution of Marangoni shear stress has been introduced coupled with a temperature difference between the fluid flow and the microfjber walls. As a result of applying this technique, the nanoparticles, which had been trapped on the microfiber walls before introducing the lechnique, have moved away from the microfiber walls allowing the fluid to flow more smoothly around the microfiber walls, relative to its motion, before applying the energy imbalance technique.

The present numerical study tends to give an engineering solution to future composites processing using nanocomposite as new modified matrix to attend multifunctional properties.
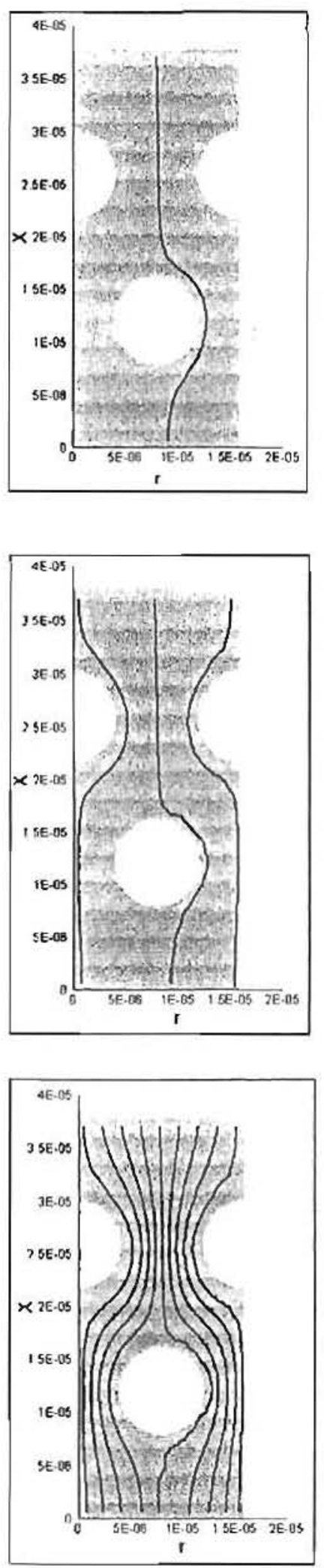

Fig. 9 Particles injection with different load ratios after introducing the energy imbalance technique 


\section{References}

1- Milton G., "The theory of composites", Cambridge University Press, New York, 2002.

2- Kim K. Utracki A., and Kamal R. "Numerical simulation of polymer nanocomposites using self-consistent meanfield model". The Journal of Chemical Physics 121: 10766-10777, 2004.

3- Chen G., Tasciuc B., and Yang G., "In Encyclopedia of Nanoscience and Nanotechnology; edited by Nalwa $\mathrm{H}^{*}$, American Scientific Publishers, Stevenson Ranch, California, vol. 7, N68, 2004.

4- Lee S., and Choi S., "Application of metalic nanoparticle suspensions in advanced cooling systems", ASME PVP 231: 227-234, 1996.

5- Xuan $Y$ and $\mathrm{Li} \mathrm{Q}_{\text {. }}$ "Heat transfer cahancement of nanofluids. Int. J. of Heat and Fluid Flow", 21: 58-64, 2000.

6- Khanafer K., Vafai K., and Lightstone $M$. "Buoyancy-driven heat transfer enhancement in a two-dimensional enclosure utilizing nanofluids". Int. J. of Heat and Mass Transfer 46: 1639-3653, 2003.

7- Elgafy A, and Lafdi K., "Effect of carbon nanofibers additives on thermal behavior of phase change materials", Carbon 43: 3067$3074,2005$.

8- Choi S. "Enhancing thernal conductivity of fluids with nanoparticles", ASME FED 231: 99-103, 1995.

9. Lee S., Choi S., Li S., and Eastman J., "Measuring thermal conductivity of fluids containing oxide nanoparticles". ASME J. Heat Transfer 121: 280-289, 1999.

10- Xuan $Y_{\text {, and Roctzel W, "Conceptions }}$ for heat transfer correlation of nanofluids". Int. J. of Heat and Mass Transfer 43: 3701$3707,2000$.

11- Eastman J., Choi S., Li S., Yu W, and Thompson L., "Anomalously increased effective thermal conductivities of ethylene glycol-based nanofluids containing copper nanoparticles", Applied Physics Letters 78: 718-720, 2001.

12- $Y_{U} W_{\text {, }}$, and Choi $S$., "The role of interfacial layers in the enhanced thermal conductivity of nanofluids: A renovated Maxwell model", J. of Nanoparticle Research 5: 167-171, 2003.

13. Mauran S. Prades P., and L'haridon F., "Heat and mass transfer in consolidated reacting beds for thermochemical systems", Heat Recovery Systems \& CHP 4: 315$319,1993$.

14. Py $X$, Olives $R$, and Mauran $S$, "Paraftin/porous graphïte-matrix composite as a high and constant power therrual storage material", Int. J. of Heat and Mass Transfer $44: 2727-2737,2001$

15- Fukai 1., Kanou M, Kodama $Y$, and Miyatake 0. . "Thermal conductivity enthancement of energy storage media using carbon fibers", Energy Conversion \& Management 4t:1543-1556, 2000.

16- Wachem B., and Almstedt A., "Methods for multiphase computational fluid dynamics", Chemical Engineering Journal 96: $81-98,2003$

17- Kleinstreuer C., "Two-phase flow", Taylor \& Frances, New York, 2003.

18. Jackson R. "Locally averaged equations of motion for a mixture of identical spherical particles and a Newtonian fluid", Chemical Engineering Science 52: 2457-2469, 1997.

19. Patankar A, and Joseph D., "Lagrangian numerical simulation of particulate flows", Int. J. of Multiphase Flow 27: 1685-1706, 2001.

20- Gidaspow D, "Multiphase flow and fluidization: continum and kinetic theory descriptions". Academic Press, San Diego, Califomis, 1994.

21- Shin S., and Juric D. "Modeling threedimensional multiphase flow using a level contour reconstruction method for front tracking without connectivity", J, of Computational Physics 180: 427-470, 2002. 
22- Ounis H., Ahmadi G., and McLaughlin B., "Brownian diffusion of submicrometer particles in the viscous sublayer", J. of Colloid and Interface Science 143: 266-277, 1991.

23- Li A., and Ahmadi G., "Computer simulation of particle deposition in the upper tracheobronchial tree", Aerosol Science and Technology 23: 202-223, 1995.

24- Li A., and Ahmadi G., "Dispersion and deposition of spherical particles from point sources in a turbulent channel flow", Aerosol Science and Technology 16: 209-226, 1992. 25- Incropera F., and DeWitt P., "Fundamentals of Heat and Mass Transfer", Fitth ed., Wiley, New York, 2002.
26- Patankar S., "Numerical heat transfer and fluid flow", Washington, DC: Hemisphere Publishing Corporation, 1980.

27- Schwartz L., "On the asymptotic analysis of surface-stress-driven thin-layer flow", J. of Engineering Mathematics 39:171-188, 2001.

28- Jiang Y., and Floryan J., "Effect of heat transfer at the interface on thermocapillary convection in adjacent phase", ASME J. Heat Transfer 125: 190-194, 2003. 